\title{
A Novel Design of Improved Hybrid Spectrum Sensing Technique for Cognitive Radio Network
}

\author{
Bhawna Ahuja \\ Department of ECE, \\ Galgotia College of Engineering and Technology \\ Greater Noida, Uttar Pradesh India
}

\author{
Gurjit Kaur \\ School of ICT, \\ Gautam Buddha University Greater Noida, Uttar \\ Pradesh \\ India
}

\begin{abstract}
The Cognitive Radio is a most authentic solution for the spectrum scarcity as there is rapid growth in wireless communication and spectrum is very limited. The main and key function of cognitive radio is to sense the idle spectrum which can be used for by secondary users in opportunistic manner. A number of techniques are proposed by various researchers. Energy Detection (ED) is the most popular technique for spectrum sensing but its performance degrades at low SNR. Spectral Covariance sensing is another effective technique which provides better detection but computationally it is more complex. It also requires higher sensing time than Energy detection. In this paper an improved hybrid spectrum sensing technique is proposed which is based on Energy detection as well as on spectral covariance based detection. Further, the performance analysis of our proposed hybrid spectrum sensing technique is evaluated. The performance is measured in terms of False alarm Probability of detection, SNR and sensing time. It has been observed that for low signal to noise ratio, where the energy detector is not reliable, the hybrid spectrum sensing technique provides improved detection. Results show that the mean detection time of the hybrid spectrum sensing technique is much lower than the Spectral covariance detection technique for most of the SNR range.
\end{abstract}

\section{Keywords}

Spectrum Scarcity, Spectrum Sensing, Energy Detection (ED, Spectral Covariance (SC), Hybrid Spectrum Sensing, Detection Performance, Sensing Time.

\section{INTRODUCTION}

Now a day the fast growth of wireless applications has increased the demand for radio spectrum dramatically. As most of the frequency bands are assigned mainly to licensed users so it becomes very difficult to find the free frequency bands to either deploy new services or enhance existing ones. (Don 2015). On the other side, results of FCC report represents that a major portion of the assigned spectrum

remains underutilized (FCC, 2002). So a technique is required to deal with this underutilized spectrum. Cognitive radio is the most promising solution for the same. It reliably senses the spectrum over a wide bandwidth, further detect the presence or absence of a primary user (licensed user) and it uses the spectrum as long as the communication does not disturb with any primary user (S. Haykin, 2005). This is because these radio channels are having lower precedence to secondary users, which not only make use of cognitive radio (CR) techniques but also ensures not to interfere with the primary users. The main objective of the cognitive radio is to reliably identify free frequency bands and this task is being performed by spectrum sensing techniques.

One of the most common techniques to sense the spectrum is Energy Detection because it is very simple but at low SNR its performance is very poor. Another efficient technique for spectrum sensing is Spectral Covariance sensing technique because It provides better detection at the cost of computationally complexity. It further needs a higher sensing time than Energy detection (Dongyue 2014). In this paper, an improved hybrid spectrum sensing technique which is derived from Energy detection and spectral covariance based detection. In the first stage, Energy detection is achieved where the threshold is compared with the received signal. If this energy is greater than the threshold level then channel is affirmed to be occupied, otherwise SCS detect the signal. If decision statistics of the SCS technique is more than the defined threshold, then channel is decided to be occupied otherwise it is declared empty and can be used by secondary users.

The rest of the paper is arranged as follows: Section II describes the basic principle of hybrid spectrum sensing along with its mathematical model. Numerical results and their analysis are discussed in section III. Conclusions drawn and outline of the future scope are given in section IV.

\section{PROPOSED HYBRID SPECTRUM SENSING TECHNIQUE}

The basic model of the proposed hybrid spectrum sensing technique is shown in fig. 1 . In the first part, radio channel is sensed using Energy detection.. If energy calculated by ED technique is greater than threshold $\lambda_{1}$ then it is assumed that channel is occupied but if energy is less than threshold then received signal is analyzed by using second spectral covariance base sensing technique.

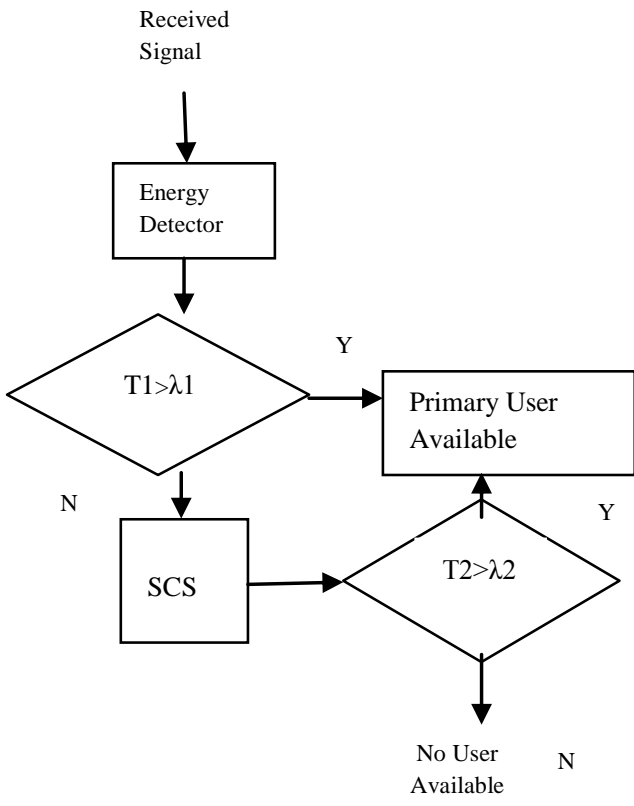

Fig 1: Proposed Hybrid Spectrum Sensing Technique 
Energy detection is selected at the first stage because of its simple construction model. It is the best possible way to detect primary signals when secondary users are unknown to priori information of the primary signal. It calculates the energy of the incoming signal over some specified interval (Urkowitz, 1967). This energy is used as test figures and compared with a threshold $\lambda$ and decides whether signal is present or absent.

The secondary user needs to take a decision between two hypotheses: either the band is vacant or occupied. Here $y(n)$ is the received vector of length $\mathrm{N}$ which consists of signal and noise. The transmitted signal of the primary user is represented as $\mathrm{x}(\mathrm{n})$, impulse response of the channel is $\mathrm{h}(\mathrm{n})$ and $w(n)$ is the response of AWGN channel with variance $\sigma^{2}$. For distinguishing the primary user signal, secondary user has to differentiate between the following two hypotheses i.e.

$\mathrm{H}_{0}: \mathrm{y}(\mathrm{n})=\mathrm{w}(\mathrm{n})$

$\mathrm{H}_{1}: \mathrm{y}(\mathrm{n})=\mathrm{x}(\mathrm{n}) \mathrm{h}(\mathrm{n})+\mathrm{w}(\mathrm{n})$

Where $\mathrm{H}_{0}$ is the null hypothesis which represents that only noise is present in the channel and the primary user is not communicating and $\mathrm{H}_{1}$ is the other hypothesis that identifies that the primary user is communicating.

The bandwidth of the signal is considered to be of W. Sampling Theorem represents that if there is energy of infinite number of terms over a duration $\mathrm{T}$, then it need approximately $2 \mathrm{u}$ samples where $\mathrm{u}=\mathrm{TW}$ is defined as time bandwidth product. (Urkowitz, 1967).

As hypothesis H0 stated that primary signal is not transmitting any data and only noise is present. So test statistic Y can be observed as the sum of the squares of 2TW standard Gaussian variates with unit variance and zero mean. The statistic $\mathrm{Y}$ precedes a central chi-square $\left(\chi^{2}\right)$ distribution with degrees of freedom as 2TW. For the second hypothesis H1, received radio signal is the combination of signal and noise. So the decision statistic $\mathrm{Y}$ will have a non-central $\chi 2$ distribution with $2 \mathrm{u}$ degrees of freedom and a non-centrality parameter $2 \gamma$

$\mathrm{Y} \sim\left\{\begin{array}{rr}\chi_{2 u}^{2}, & \mathrm{H}_{0} \\ \chi_{2 u}^{2},(2 \gamma) & \mathrm{H}_{1}\end{array}\right.$

Probability of false alarm can be calculated by (Digham, 2003)

$\operatorname{Pf}=\operatorname{Pr}(E>\lambda \mid H 0)=\int_{\lambda}^{\infty} f_{x^{2}}(y) d y=\frac{\Gamma(u, \lambda / 2)}{\Gamma(u)}$

Similarly

$\operatorname{Pd}=\operatorname{Pr}(E>\lambda \mid H 1)=Q_{u}(\sqrt{2 \gamma}, \sqrt{\lambda})$

Where $\gamma$ is the signal to noise ratio .Г(.) is complete and $Г(.,$. is incomplete gamma functions . Generalized Marcum Qfunction is represented as $\mathrm{Qu}$ and $\mathrm{u}=\mathrm{TW}$. For $\mathrm{u}>125$ it assumes that the sample number is quite large and all the sampled signals are not depending on each other. So the decision statistic could be concluded which conform the Gaussian distribution according to the Central-Limit Theorem (Urkowitz, 1967).

For the second spectrum sensing hybrid stage, a spectral covariance detector is invoked which will give better performance than the energy detector mainly for low SNR. Here periodogram estimation is used when spectrum of the received signal is obtained and its correlation is calculated by using the sample covariance matrix. Usually the primary user has a unique non-flat spectrum which makes it extremely correlated but the noise is entirely uncorrelated because the spectrum is flat. The relative correlation can be calculated by comparing the auto covariance of the spectrum with the total covariance.

If the spectral correlation is greater than the said threshold, signal is detected. Firstly SCS technique down converts the received signal to a baseband complex signal. Then a low pass filter is used which downsample the baseband signal which further helps by averaging out zero mean noise. It also prevent aliasing and suppresses noise power in stop band. The spectrogram is computed by analysing the squared magnitude of its Short Time Fourier transform (STFT). Then the components are selected near DC for having reduced spectrogram matrix $\mathrm{M}$. The sample covariance of $\mathrm{M}$ can be calculated as $\mathrm{C}=\operatorname{cov}(\mathrm{M})$. After calculating covariance test statistic $\mathrm{T}_{2}$ is computed. At last $\mathrm{T}$ is compared with the decision threshold and decided if it's greater than the said threshold value.

\section{PERFORMANCE ANALYSIS OF PROPOSED HYBRID SPECTRUM SENSING TECHNIQUE}

The performance of proposed hybrid spectrum sensing technique is analyzed as follows:

\subsection{Sensing Performance}

In this hybrid spectrum sensing technique, a false alarm appears if, under $\mathrm{H} 0$,

$$
\begin{aligned}
& \mathrm{T}_{1}>\lambda_{1}, \text { or } \\
& \mathrm{T}_{2}>\lambda_{2} \text { given that } \mathrm{T}_{1} \leq \lambda .
\end{aligned}
$$

Similarly, a right detection appears if, under H1. So the overall probability of detection and false alarm of the hybrid energy and spectral covariance detector is given by the following formula

$$
\begin{aligned}
& P_{d, E S}=P_{d, E}+P_{d, s c s}\left(1-P_{d, E}\right) \\
& P_{f, E S}=P_{f, E}+P_{f, s c s}\left(1-P_{f}, E\right)
\end{aligned}
$$

\subsection{Mean Detection Time}

The mean detection time of the hybrid spectrum sensing technique has two terms

$$
\bar{T}=\overline{T_{E D}}+\overline{T_{S C S}}
$$

Where $\overline{T_{E D}}$ is sensing time of the energy detection technique and it is given as $\overline{T_{E D}}=\mathrm{LT}_{\mathrm{ED} \text { with }} T_{E D}=\frac{N_{E D}}{2 W}$. Here $\mathrm{N}_{\mathrm{ED}}$ is number of samples required in energy detection and channel bandwidth is represented as $\mathrm{W}$. The number of channels to be sensed are represented by L. The sensing time of SCS is $\overline{T_{S C S}}$ which can be derived by following formula

$$
\overline{T_{S C S}}=E[K] T_{\mathrm{SCS}}
$$

Here $\overline{T_{S C S}}$ is the sensing time for each channel. The number of samples required for Spectral covariance detection is $\mathrm{N}_{\mathrm{SCS}}$. Random variable follows a binomial distribution with parameters L. $P_{S C S}$ is the probability of channel sensing calculated by the SCS detector. It is shown that $\mathrm{P}_{\mathrm{SCS}}$ i.e Probability of activation of the second stage in conventional two stage detector is given as

$$
\mathrm{P}_{\mathrm{SCS}}=\mathrm{P}\left(\mathrm{H}_{0}\right)\left(1-\mathrm{P}_{\mathrm{f}, \mathrm{ED}}\right)+\mathrm{P}\left(\mathrm{H}_{1}\right)\left(1-\mathrm{P}_{\mathrm{d}, \mathrm{ED}}\right)
$$

Where $\mathrm{P}(\mathrm{H} 0)$ is the probability of the channel being vacant and $\mathrm{P}(\mathrm{H} 1)$ is the probability of the channel occupacy by the primary user. Thus if TED is the time taken by the ED in the first stage and TSCS is the time taken by the second stage used for secondary detection i.e SCS, then the mean detection time of the proposed hybrid spectrum sensing technique is calculated as 
$\left.\overline{\mathrm{T}}=\frac{\mathrm{L}}{\mathrm{W}}\left\{\mathrm{N}_{\mathrm{ED}}+\left[\mathrm{P}\left(\mathrm{H}_{0}\right)\left(1-\mathrm{P}_{\mathrm{f}, \mathrm{ED}}\right)+\mathrm{P}\left(\mathrm{H}_{1}\right)\left(1-\mathrm{P}_{\mathrm{d}, \mathrm{ED}}\right)\right] \mathrm{N}_{\mathrm{SCS}}\right]\right\}$

It implies that

$$
\overline{\mathrm{T}}=\mathrm{L}\left(\mathrm{T}_{\mathrm{ED}}+\mathrm{P}_{\mathrm{SCS}} \mathrm{T}_{\mathrm{SCS}}\right)
$$

\section{RESULTS AND ANALYSIS}

In this section the performance of the proposed hybrid spectrum sensing technique with the existing techniques i.e. only energy detection or SCS detection is compared. The performance is evaluated using curves of $\mathrm{P}_{\mathrm{d}}$ Vs SNR for different values of probability of false alarm $\left(\mathrm{P}_{\mathrm{f}}\right)$ and sensing time. ROC curves under different value of SNR are also discussed to assess the performance of energy detector. Assume signal is simulated as a primary signal with $200 \mathrm{KHz}$ bandwidth. The sampling frequency was fixed at $1000 \mathrm{KHz}$ with $\mathrm{fm}$ and $\mathrm{fc}$ as $100 \mathrm{KHz}$ and $3 \mathrm{MHz}$ respectively where $\mathrm{fm}$ is modulating Frequency and fc is carrier frequency. Spectrum of signal is shown in Fig 2 . In order to achieve the convergence, 1000 simulation runs were used for the parameters.
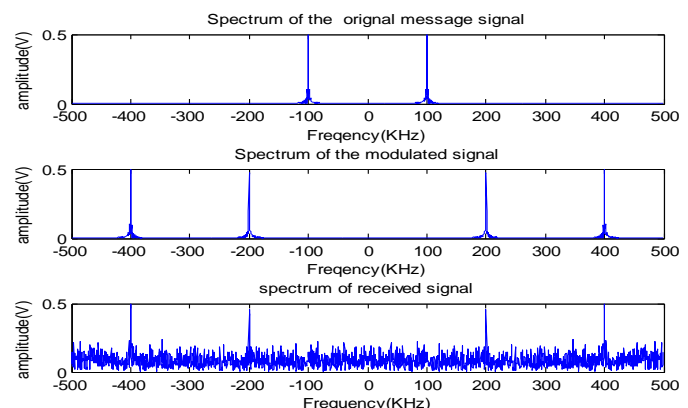

Fig 2: Spectrum of simulated signal

As shown in fig. 3 and fig 4 probability of detection is plotted as a function of SNR for Energy Detector, SCS detector and Two stage Detector for given $\mathrm{P}_{\mathrm{f}}=0.01$ and $\mathrm{P}_{\mathrm{f}}=0.1$. for sensing time $\mathrm{T}_{\mathrm{ED}}=1 \mathrm{~ms}$ and . Fig. 3 and 4 represents the detection performance versus signal to noise ratio for the three sensing techniques for sensing times $\mathrm{T}_{1}=2 \mathrm{~ms}$ and $\mathrm{T}_{2}=8 \mathrm{~ms}$. From the results it is concluded that for an SNR that is less than $-12 \mathrm{~dB}$, the hybrid spectrum sensing technique performs better than either energy detection or SCS detection.

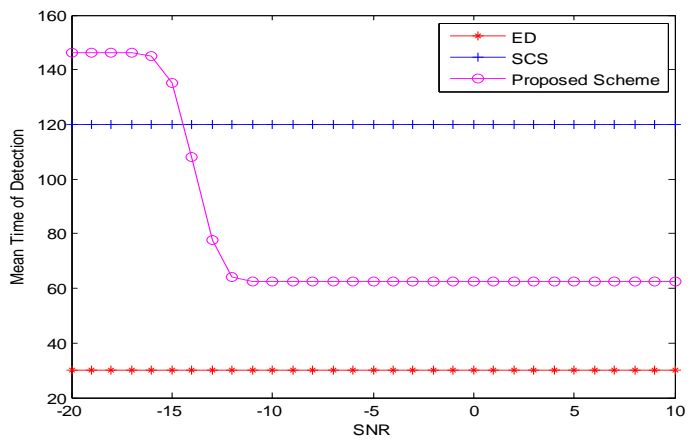

Fig 3: Sensing Time for $P\left(H_{0}\right)=0.3$

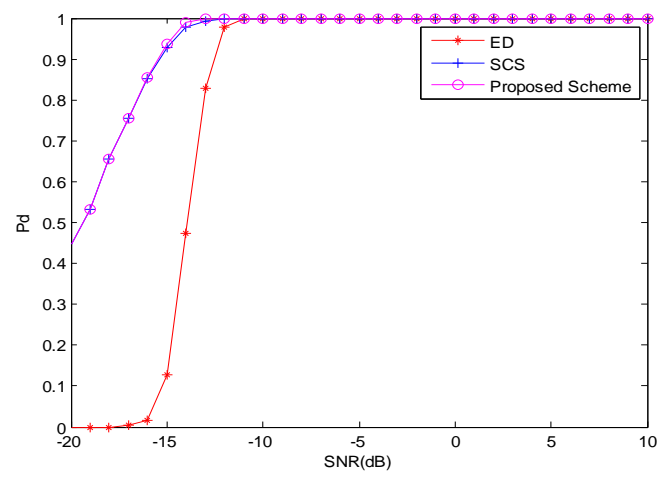

Fig 4: $P_{d}$ Vs SNR for $P f=0.1$

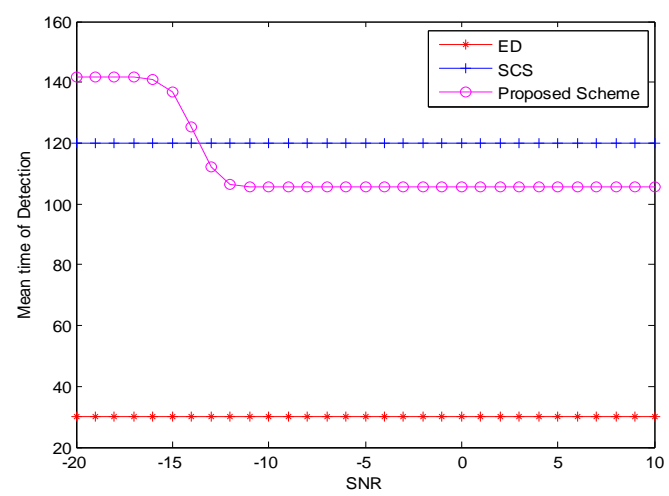

Fig 5: Sensing Time for $P\left(H_{0}\right)=0.7$

Fig 5 represents the detection time for the proposed hybrid spectrum sensing technique compared to the energy and SCS detectors for different SNRs. For $\mathrm{P}\left(\mathrm{H}_{0}\right)=0.2$ and 0.8 respectively. For $\mathrm{P}\left(\mathrm{H}_{0}\right)=0.2$, hybrid spectrum sensing outperforms the SCS detector in terms of mean detection time as well as detection performance when SNR is less than $<-12$ $\mathrm{db}$. For $\mathrm{P}\left(\mathrm{H}_{0}\right)=0.8$, PSCS is higher, two-stage sensing does not always have a smaller mean detection time than the SCS detector.

\section{CONCLUSIONS}

This paper reported the performance analysis of hybrid spectrum sensing technique which is based on Energy detection and spectral covariance sensing. Probability of detection, SNR and sensing time are used as performance metrics. Through numerical results obtained via computer simulation and analytical formulation, it is observed that at low SNR, where the energy detector is not reliable, the proposed hybrid spectrum sensing provides improved detection. Furthermore, results shows that the mean detection time of the proposed spectrum sensing is much lower than the Spectral covariance detection technique for most of the SNR range. Hence, proposed technique provides a good tradeoff between performance and detection time. In future some computational techniques can be applied along with the proposed technique to improve further results.

\section{REFERENCES}

[1] I. F. Akyildiz, B. F. Lo, and R. Balakrishnan(2011), "Cooperative Spectrum Sensing in Cognitive Radio Networks: A Survey," Physical Communication (Elsevier) Journal, vol. 4, no. 1, pp. 40-62

[2] H. Urkowitz (1967), "Energy detection of unknown deterministic signals," Proc. IEEE, vol. 55, pp. 523-531, Apr. 1967. 
[3] FCC (2003), "Notice of proposed rule making and order".ET Docket No 03-222,2003

[4] F. F. Digham, M. S. Alouini, and M. K. Simon, (2003) "On the energy detection of unknown signals over fading channels," in Proc. IEEE Int. conf. Commun., , vol. 5, pp. 3575-3579.

[5] R. Tandra and A. Sahai (2008), "SNR walls for signal detection," IEEE Journal of Selected Topics in Signal Processing, vol. 2, no. 1, pp.4-17

[6] Zhuan Ye, Gokhan Memik, John Grossietsch(2008), "Energy Detection using Estimated Noise Variance for Spectrum Sesning in Cognitive Radio Networks", Proceedings of WCNC 2008, pp. 2230-2236

[7] S.Maleki, A. Pandharipande, andG. Leus, "Two-stage spectrum sensing for cognitive radios," in Proceedings of the IEEE International Conference on Acoustics, Speech and Signal Processing (ICASSP '10), pp. 2946-2949, March 2010.
[8] Lee, W.Y. and Akhilidiz, I.F. (2012) Spectrum Aware Mobility Management in Cognitive Radio Cellular Networks. IEEE Transactions on Mobile Computing, 11, 529-542.

http://dx.doi.org/10.1109/TMC.2011.69

[9] Don Jun Lee (2015) Adaptive Random Access for Cooperative Spectrum Sensing in Cognitive Radio Networks, Wireless Communications , IEEE Transactions on Wireless Communications Volume: 14, Issue: 2 ,DOI: 10.1109/TWC.2014.2360857 , Page(s): $831-840$

[10] Dongyue Xue ; Ekici, E. ; Vuran, M.C (2014) Cooperative Spectrum Sensing in Cognitive Radio Networks Using Multidimensional Correlations , IEEE Transactions on Wireless Communications, Volume: 13 , Issue: 4 , Page(s): 1832 - 1843. 